\title{
Ergonomic Risk Assessment using Cornell Musculoskeletal Discomfort Questionnaire in a Grocery Store
}

\section{Erman Çakıt* \\ Department of Industrial Engineering, Gazi University, Turkey}

*Corresponding author: Erman Çakıt, Department of Industrial Engineering, Gazi University, Turkey, Tel: +90 312582 3809; Email: ecakit@gazi.edu.tr / ecakit@gmail.com

\section{Research Article}

Volume 3 Issue 6

Received Date: November 25, 2019

Published Date: December 13, 2019

DOI: $10.23880 /$ eoij-16000222

\section{Abstract}

In most grocery stores in Turkey, workers are standing while their shift. They are also subjected to repetitive tasks and awkward postures. The aim of this cross-sectional analysis was to explain the prevalence of work-related symptoms of musculoskeletal in grocery shops. The study participants $(n=10)$ included supermarket staff from five separate grocery stores in a medium-sized grocery chain. In particular, the perceived pain of each worker was determined using Cornell Musculoskeletal Discomfort Questionnaire (CMDQ). The results obtained using CMDQ work positions are in need of change. Furthermore, the results can be used for recommendations to improve ergonomics factors of the tasks in form of a guideline or general statements presenting recommendations for mitigating risks.

Keywords: Ergonomics Assessment; CMDQ; Musculoskeletal Disorders; Grocery

Abbreviations: CMDQ: Cornell Musculoskeletal Discomfort Questionnaire; MSDs: Musculoskeletal Disorders; DC: Distribution Center.

\section{Introduction}

The complaints among grocery workers about musculoskeletal disorders (MSDs) have been increasing in the last decade and have become a major health issue. Work-related MSDs may cause disability workers.

WMSDs account for the highest proportion of workers' compensation claims and permanent disability. [1]. WMSDs are types of disorders affecting various body parts that are associated with movement, including the upper limbs, lower limbs, and back. These affect the different structures of the body such as tendons, joints, muscles, and nerves and are primarily caused or aggravated by work-related activities [2].

Other risk factors were proposed that were of special interest, such as joint positions such as cramped positions, extreme positions and steep forward bending of the head. Therefore, the problems can be aggravated by high repetitivity, high pressure, high static muscles and joint load [3]. The frequency of WMSDs can be determined by the workers' compensation claims among any working population. A higher incidence or prevalence of these workers in a workplace population indicates that they are more vulnerable to the physical risk factors. Nonetheless, incidence estimates require large employees to be sampled. Moreover, the prevalence of WMSDs in compensation claims are frequently underestimated [4]. 


\section{Ergonomics International Journal}

Studies into musculoskeletal symptoms may provide closely related estimates of WMSD in different body areas. The study aimed at preventing musculoskeletal symptoms related to work in grocery store workers from various departments and determining the risk factors with the use of CMDQ. In warehousing / grocery distribution center (DC) activities, a small amount of research has been conducted specifically addressing ergonomics. NIOSH researchers performed a supermarket warehouse risk study in which the incidence of back injuries was 16 per 100 employees. They considered lift loads exceeding acceptable limits and levels, locations from or to which objects are selected or put to be too low, too high, or too deep, and work times exceeding 8 hours to be troubling [5]. In a previous study on Wholesale Selectors [6], the development of storage systems placed excessive reach positions that would cause half male and 90 percent of females to over exercise on the job, based on biomechanical modeled jobs. The researchers identified factors in many aspects of the work system in an analysis of the problematic working factors for inventors of warehousing surfaces that made the job less productive and dangerous [7].

Although WMSDs affect all sectors of the working population, there are particularly high MSD rates among grocery employees. During the period 1997-2005, grocery stores in Washington State had a level of musculoskeletal injury 1.8 times higher than the state average and 5th in all industries' compensable upper extremity WMSDs [8]. Like shoulder, rotator cuff syndrome, wrist tendonitis, carpal tunnel syndrome, and back disorders like sciatica, grocery jobs ranked in the top 25 injury occupations. Health Assessment \& Research for Prevention (SHARP) concluding that grocery work has become one of the "top twelve industries in need of focused research and prevention efforts."

Most of the research carried out in grocery workers focused on repeat hand movements by cashiers and the checkout station layout [9-13]. Workers in grocery markets are, therefore, impaired by physiological conditions and WMSDs pose risk factors. Types involve heavy carrying to the shelf from the warehouse. High force occurs while heavy product is lifted. And, it affects shoulder position.

The rest of the paper is as follows described. Section 3 details the methodological steps, including participants, procedure, CMDQ, and study steps. Section 4 addresses the results. Ultimately, in sections 5 and 6, respectively, the discussion and conclusions are presented.

\section{Methodology}

\section{Participants}

There were 5 healthy male and 5 healthy female from different departments among the study groups. The population was between the ages of 18 and 49.The ergonomic risk analysis using CMDQ for the grocery practiced in the well-known grocery store in Ankara, Turkey. The mean values and the standard differences between age, height and weight of the individuals were 33 $\pm 10,58$ years, $167 \pm 13,02 \mathrm{~cm}$, and $64,1 \pm 9,31 \mathrm{~kg}$, respectively (Table 1). The study informed the participants and each of them indicated their willingness to participate with a participatory consent form. The participants received and read the information letter before responding to questionnaire. None of the participants reported musculoskeletal problems during the study.

\begin{tabular}{|c|c|c|c|c|}
\hline & Mean & SD & Minimum & Maximum \\
\hline Age & 33 & 10.58 & 18 & 49 \\
\hline Height (cm) & 167 & 13.02 & 150 & 183 \\
\hline Weight (kg) & 64.1 & 9.31 & 50 & 80 \\
\hline Work experience (years) & 6.8 & 5.62 & 1 & 20 \\
\hline Working hours & 9.7 & 1.13 & 8 & 12 \\
\hline
\end{tabular}

Table 1: Demographic Statistics.

\section{Procedure}

The subjects were explained the questionnaire and the CMDQ was used in order to gather data. The questionnaires began with demographic questions regarding gender, age, years of experience, and number of hours per shift. Subjects were allowed to spend as much time as they needed to answer each question. The researcher answered any questions that arose during the administration of the questionnaire but provided no assistance on content. The subjects were not allowed to consult other respondents or discuss with them before or
Erman Çakıt. Ergonomic Risk Assessment using Cornell Musculoskeletal Discomfort Questionnaire in a Grocery Store. Ergonomics Int J 2019, 3(6): 000222. 


\section{Ergonomics International Journal}

during the study. This was achieved by having all the subjects filling the questionnaires at the same time in different cubicles.

\section{Cornell Musculoskeletal Discomfort Questionnaire}

The Cornell Musculoskeletal Discomfort Questionnaire (CMDQ) was used to assess discomfort. Professor Alan Hedge and Ergonomics students of the Cornell University developed a well-designed data collection tool named
CMDQ [14]. The CMDQ is a 54-item questionnaire that includes a body chart and questions about musculoskeletal ache, pain or discomfort occurrence in 20 parts of the body over the past week. (Figure 1). It has been used in working groups such as healthcare provider and machine operator in the evaluation of musculoskeletal pain. The musculoskeletal discomfort score was calculated in accordance to the CMDQ scoring guidelines for the determination of the rate of discomfort and the quantification of the discomfort level.

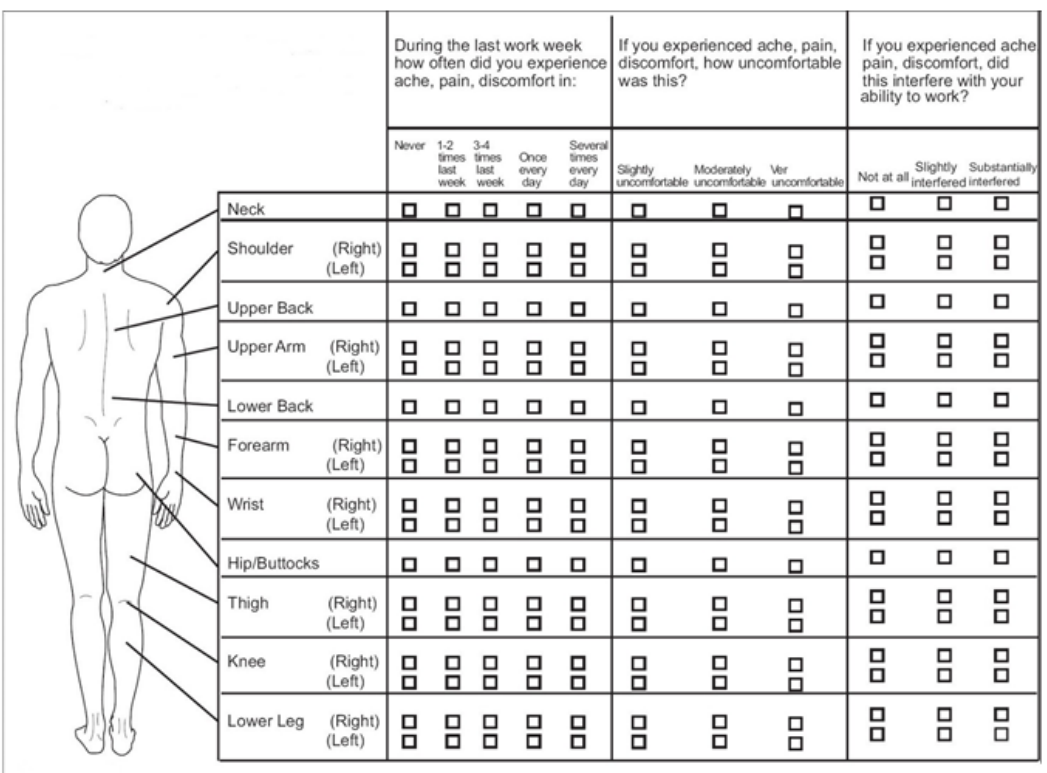

Figure 1: Cornell Musculoskeletal Discomfort Questionnaire, male version.

The assessment of musculoskeletal discomfort was presented as follows. Firstly, the level of discomfort recorded during the survey by the operators was calculated as: "Never (0), 1 or 2 times/week (1.5), 3 or 4 times/week (3.5), every day (5), or several times every day (10)". In order to reach the weighted musculoskeletal discomfort level, the result will then be multiplied by the severity rate ("slightly uncomfortable $=1$, moderately uncomfortable $=2$, very uncomfortable $=3 "$ ) and interference rating ("Not at all $=1$, slightly interfered $=2$, substantially interfered $=3 "$ "). The most severe cases were thus described.

\section{Study Steps}

The study has been divided into three phases. The first phase was preparatory and included:
- Informing the participant about the purpose, method and risk assessment

- Obtaining informed consent of the individual

- Recording subject age, height, weight, gender, educational situation, work experience, working hours in a day, the grocery they work.

The Cornell assessment portion of the study started after the paperwork was finished. The researchers first demonstrated the procedure to ensure that participants understood what was needed. The process involved CMDQ:

- Participant was given brief introduction and requested the complete CMDQ independently

- Each participant was interwoven about clarity and understandability of the questionary.
Erman Cakit. Ergonomic Risk Assessment using Cornell Musculoskeletal Discomfort Questionnaire in a Grocery Store. Ergonomics Int J 2019, 3(6): 000222. 


\section{Ergonomics International Journal}

\section{Results and Discussion}

The results of CMDQ reported total body discomfort and high impact long working hours and unfavorable working conditions. To evaluate concurrent validity, the Cornell musculoskeletal discomfort questionnaire was also employed. The studies of musculoskeletal disorders consider International Musculoskeletal Disorder (MSD) Questionnaire as an essential feature [15]. Since the CMDQ examines discomfort levels according to frequency and severity and it also analyzes the rate at which work performances of individual are negatively influenced and this statement was mentioned by previous recent studies $[16,17]$.

According to the total discomfort score of CMDQ (Table 2), it was concluded that grocery workers felt discomfort mostly in the upper back $(17,29 \%)$, lower back $(13.23 \%)$ and the right shoulder $(9,52 \%)$, while it was less pronounced in the left foot $(0.72 \%)$, right foot $(0,72 \%)$ and left thigh $(1.62 \%)$. The low and the upper back were anatomical areas with the highest prevalence of symptoms, similar to other studies conducted by grocery workers $[10,13,18]$.

\begin{tabular}{|c|c|c|c|c|c|}
\hline Body parts referred to in the questionnaire & Frequency & Discomfort & Interference & Discomfort score & \% \\
\hline Upper back & 57 & 22 & 20 & 25080 & 17,292 \\
\hline Lower back & 50,5 & 20 & 19 & 19190 & 13,231 \\
\hline Shoulder_R & 47 & 21 & 14 & 13818 & 95,273 \\
\hline Neck & 50,5 & 18 & 14 & 12726 & 87,744 \\
\hline Shoulder_L & 45 & 19 & 14 & 11970 & 82,532 \\
\hline Upper arm_R & 31,50 & 17 & 15 & 8032,5 & 55,383 \\
\hline Upper arm_L & 31,50 & 17 & 15 & 8032,5 & 55,383 \\
\hline Lower leg_R & 25 & 15 & 15 & 5625 & 38,784 \\
\hline Forearm_L & 29,5 & 14 & 13 & 5369 & 37,019 \\
\hline Forearm_R & 27,5 & 15 & 13 & 5362,5 & 36,974 \\
\hline Lower leg_L & 25 & 14 & 15 & 5250 & 36,198 \\
\hline Wrist_R & 22,5 & 14 & 13 & 4095 & 28,234 \\
\hline Wrist_L & 20,5 & 14 & 13 & 3731 & 25,725 \\
\hline Hip & 18,5 & 16 & 12 & 3552 & 24,491 \\
\hline Knee_R & 16,5 & 14 & 15 & 3465 & 23,891 \\
\hline Knee_L & 13 & 16 & 14 & 2912 & 20,078 \\
\hline Thigh_R & 16,5 & 11 & 13 & 2359,5 & 16,268 \\
\hline Thigh_L & 16,5 & 11 & 13 & 2359,5 & 16,268 \\
\hline Foot_R & 13 & 9 & 9 & 1053 & 0,726 \\
\hline Foot_L & 13 & 9 & 9 & 1053 & 0,726 \\
\hline
\end{tabular}

Table 2: Total discomfort score felt by the grocery workers.

Differences between studies over the years of industry work and demographic differences like the gender distribution of samples may cause a variance in the prevalence of studies. In the present study, the results could have been affected by some limitations. Firstly, it could have been possible to use other forms of exposure assessment. Violante and colleagues [13] used the lifting equation of NIOSH to estimate a low, moderate, and high risk of heavy lifting in a study of low back injuries among grocery employees, for example.

\section{Conclusion}

Musculoskeletal discomforts and symptoms related to work are largely due to psychological (stress, cognitive load, etc) and organizational (unsuitable working-rest period, lack of work-enrichment etc.) factors in the work environment (static and bad postures, repetition of movements, non-ergonomic workstation configuration, etc.). It is important to improve working conditions, design and layout of workplaces by organizations and successful ergonomic interventions in the workplace. An assessment of the workplace, monitoring of the associated risk factors, medical management and education are important for preventing and eliminating WMSDs. The study showed that in the upper and lower back, the feeling of discomfort felt subjectively by grocery workers was higher. The relationship between musculoskeletal discomfort and productivity needs to be further studied. The result of this analysis can therefore

Erman Çakıt. Ergonomic Risk Assessment using Cornell 


\section{Ergonomics International Journal}

be used to further assess the impact of MSD on productivity.

\section{References}

1. Bureau of Labor Statistics (2013) Nonfatal Occupational Injuries and Illnesses Requiring Days Away From Work. Economic News Release.

2. Nunes IL, Bush PM (2012) Work-related musculoskeletal disorders assessment and prevention. Ergonomics-A Systems Approach.

3. Stock SR (1991) Workplace ergonomic factors and the development of musculoskeletal disorders of the neck and upper limbs: A meta-analysis. American journal of industrial medicine 19(1): 87-107.

4. Major ME, Vézina N (2015) Analysis of worker strategies: a comprehensive understanding for the prevention of work related musculoskeletal disorders. International Journal of Industrial Ergonomics 48: 149-157.

5. Putz-Anderson V, Waters TR, Baron S, Hanley K (1993) Big Bear grocery warehouse, Columbus, OH. HETA 91-405-2340, National Institute for Occupational Safety and Health.

6. Garg A (1986) Biomechanical and ergonomic stresses in warehouse operations. IIE transactions 18(3): 246250.

7. St-Vincent M, Denis D, Imbeau D, Laberge M (2005) Work factors affecting manual materials handling in a warehouse superstore. International Journal of Industrial Ergonomics 35(1): 33-46.

8. Silverstein B, Adams D (2007) Work-related musculoskeletal disorders of the neck, back, and upper extremity in Washington State, 19972005. Olympia: Safety and Health Assessment and Research for Prevention, Department of Labor and Industries, Washington.

9. Carrasco C, Coleman N, Healey S, Lusted M (1995) Packing products for customers: an ergonomics evaluation of three supermarket checkouts. Applied ergonomics 26(2): 101-108.
10. Forcier L, Lapointe C, Lortie M, Buckle P, Kuorinka I, et al. (2008) Supermarket workers: Their work and their health, particularly their self-reported musculoskeletal problems and compensable injuries. Work 30(4): 493-510.

11. Rodacki ALF, Vieira JEA, Okimoto MLL, Fowler NE, Rodacki CDLN (2006) The effect of handling products of different weights on trunk kinematics of supermarket cashiers. International journal of industrial ergonomics 36(2): 129-134.

12. Spielholz P, Howard N, Carcamo E, Coulter B (2008) Field evaluation of a new grocery checkstand design. Applied ergonomics 39(1): 87-91.

13. Violante FS, Graziosi F, Bonfiglioli R, Curti S, Mattioli S (2005) Relations between occupational, psychosocial and individual factors and three different categories of back disorder among supermarket workers. International archives of occupational and environmental health 78(8): 613-624.

14. Hedge A, Morimoto S, Mccrobie D (1999) Effects of keyboard tray geometry on upper body posture and comfort. Ergonomics 42(10): 1333-1349.

15. Erdinc 0, Hot K, Ozkaya M (2011) Turkish version of the Cornell Musculoskeletal Discomfort Questionnaire: Cross-cultural adaptation and validation. Work 39(3): 251-260.

16. Habibi E, Taheri MR, Hasanzadeh A (2015) Relationship between mental workload and musculoskeletal disorders among Alzahra Hospital nurses. Iranian journal of nursing and midwifery research 20(1): 1-6.

17. Sethi J, Sandhu JS, Imbanathan V (2011) Effect of Body Mass Index on work related musculoskeletal discomfort and occupational stress of computer workers in a developed ergonomic setup. Sports Medicine, Arthroscopy, Rehabilitation, Therapy \& Technology 3(1): 22.

18. Anthony Ryan G (1989) The prevalence of musculoskeletal symptoms in supermarket workers. Ergonomics 32(4): 359-371. 